\title{
Review: nurse practitioner primary care improves patient satisfaction and quality of care with no difference in health outcomes
}

\author{
Horrocks S, Anderson E, Salisbury C. Systematic review of whether nurse practitioners working in primary care can \\ provide equivalent care to doctors. BMJ 2002 Apr 6;324:819-23.

\section{QUESTION: Do nurse practitioners (NPS) provide equivalent primary care at first point of contact to patients with undifferentiated health problems when compared with physicians?}

\section{Data sources}

Published and unpublished studies were identified by searching Medline (1966-2001), EMBASE/Excerpta Medica (1980-2001), CINAHL (1982-2001), Science Citation Index, Database of Abstracts of Reviews of Effectiveness, National Research Register, Cochrane Controlled Trials Register, and the Cochrane Effective Practice and Organisation of Care Group specialist register; contacting authors of included studies, NP organisations, and educational training centres; and handsearching recent relevant journals, bibliographies, and reference lists.

\section{Study selection}

Studies in any language were selected if they were randomised controlled trials (RCTs) or prospective observational studies from developed countries that compared NPs and physicians working in the same way as concurrent controls; nurses provided care at first point of contact, made an initial assessment, and managed patients autonomously; nurses provided primary care at first point of contact for unselected patients; care was for undiagnosed patients with undifferentiated health problems; and if studies reported $\geq 1$ of the main outcomes.

\section{Data extraction}

2 reviewers independently extracted data on patients, setting, nurses (including qualification level) and physicians, study quality, and main outcomes (patient satisfaction, health status, process of care, quality of care, and health service costs).

\section{Main results}

34 studies (11 RCTs and 23 observational studies) met the selection criteria. Only data from RCTs are reported here; the findings of observational studies were consistent with those from RCTs, except for investigation outcomes. 9 RCTs reported on patient satisfaction. Meta-analysis of 5 studies reporting continuous data showed that patients were more satisfied with NPs than with physicians (standardised mean difference $0.27,95 \%$ CI 0.07 to 0.47 ), whereas meta-analysis of 3 RCTs reporting dichotomous data found no difference between provider groups $(p=0.4) .7$ RCTs (not meta-analysed because of heterogeneity) showed no difference between provider groups for health status. Up to 6 RCTs reported on process measures and found that NPs had longer consultations than physicians (5 studies, weighted mean difference $3.67 \mathrm{~min}$, CI 2.05 to 5.29) and did more investigations (odds ratio 1.22, CI 1.02 to 1.46 ). The provider groups did not differ for prescriptions, return consultations, or referrals. 6 RCTs (not meta-analysed because of heterogeneity) generally found better quality of care with NPs than with physicians. 5 studies provided data about costs but were inadequately powered for economic analysis.

\section{Conclusions}

Nurse practitioner primary care at first point of contact improves patient satisfaction and quality of care compared with physician care, with no difference in health outcomes. Nurse practitioners also had longer consultation times and did more investigations.
Source of funding:

South and West

Research and

Development

Directorate.

For correspondence: Dr C Salisbury,

Division of Primary

Health Care, University

of Bristol, Bristol, UK.

c.salisbury@bristol.ac.uk

\section{COMMENTARY}

Systematic reviews comparing primary health care delivered by NPs with that delivered by physicians are dated. ${ }^{12}$ The systematic review by Horrocks et al includes studies done up to and including 2001 and provides a current consolidation of research evidence on this important topic. The strengths of this review include the extensive search for published and unpublished studies; critical appraisal of studies using established criteria; contact with authors of included studies for additional information; independent extraction of data by 2 reviewers; separate analysis of data from RCTs; and consideration of heterogeneity among studies.

The weaknesses of this review include the broad definition of the term "nurse practitioner". Studies were included if nurses were the first point of contact, completed the initial assessment, and autonomously managed patient care, even when the nurses were not described as NPs. Settings varied and included general practice, out of hours centres, walk in centres, and emergency departments. To their credit, the authors used sensitivity analysis to examine the effect of including or excluding studies in which it was unclear whether the nurse was a NP. The reviewers were unable to complete an economic analysis because the studies used different approaches to valuing of resources and were not sufficiently powered. Although the results show that NPs did more investigations than physicians, the appropriateness of the investigations was not evaluated. This information is important to determine whether the additional investigations were of benefit.

It is important to note that these trials compared NPs with physicians in areas where their scope of practice overlapped. A large part of the NP's role does not overlap with that of the physician but rather is complementary. It is time to steer away from the comparison of these 2 providers and move toward an evaluation of care provided by a team that includes NPs and one that does not.

This systematic review lends strength to the argument that better utilisation of NPs, working within their scope of practice, could improve access to primary health care for the general population. Further research is needed in settings where NPs have not traditionally worked, such as long term and acute care settings.

Faith C Donald, RN(EC), MS(CHN), PHCNP Assistant Professor Ryerson University School of Nursing Toronto, Ontario, Canada

Corinne McCurdy, RN(EC), BScN Primary Care Nurse Practitioner Heart Transplant Program Toronto General Hospital Toronto, Ontario, Canada

1 Sox HC Jr. Quality of patient care by nurse practitioners and physician's assistants: a tenyear perspective. Ann Intern Med 1979;91:459-68.

Brown SA, Grimes DE. A meta-analysis of nurse practitioners and nurse midwives in primary care. Nurs Res 1995;44:332-9. 